\title{
Embedding a Graph in the Grid of a Surface with the Minimum Number of Bends Is NP-hard
}

\author{
M. A. Garrido and A. Márquez \\ Dept. Matemática Aplicada I, Universidad de Sevilla, 41012-Sevilla (Spain) \\ email: vizuete@cica.es and almar@cica.es
}

\begin{abstract}
This paper is devoted to the study of graph embeddings in the grid of non-planar surfaces. We provide an adequate model for those embeddings and we study the complexity of minimizing the number of bends. In particular, we prove that testing whether a graph admits a rectilinear (without bends) embedding essentially equivalent to a given embedding, and that given a graph, testing if there exists a surface such that the graph admits a rectilinear embedding in that surface are NPcomplete problems and hence the corresponding optimization problems are NP-hard.
\end{abstract}

\section{Introduction}

Drawings of graphs on the rectilinear grid have been studied because of their applications in VLSI planning. In this context, three important optimization subtasks related to theoretical problems in Graph Theory arise in VLSI design

- To minimize the number of holes to be made in the board in order to avoid inappropiate intersection.

- To minimize the number of bends.

- To minimize the area.

This paper is related to diverse aspects of the second problem. The first task is one of the most important problems in Topological Graph Theory, where it is known as the genus of a graph, and, in $1990 \mathrm{C}$. Thomassen proved that this problem is NP-hard [8]. Nonetheless, several heuristics have been presented obtaining good aproximations to the optimal solution (see [6]). The other two problems are NP-hard problems even if there exists no fixed embedding of the graph (see [4] and [1]). In practice, the heuristics mentioned above provide actual embeddings of the graphs in certain surfaces. Thus, it remains as an open question to find a polynomial algorithm providing the minimum number of bends when a fixed embedding in a surface is given. In this sense, Tamassia proves that if the embedding is in the plane that algorithm exists [7], but nothing is known in other surfaces. And, actually in most practical situations, the graph that modelizes the design is non-planar. In this paper, we establish the basis to the study of grid drawings of graphs in orientable surfaces defining a good model (a grid in each surface of genus $n$ ) that allows to considerate the same topics as in the plane, and we prove that the two following related problems are NP-complete: 
- Testing whether a graph admits a rectilinear (without bends) embedding essentially equivalent to a given embedding.

- Given a graph, testing if there exist a surface such that the graph admits a rectilinear embedding in that surface.

Hence, the corresponding bend-minimization problems are NP-hard. In this paper, we assume standard concepts on NP-completeness [3].

\section{A model to study grid embeddings in non-planar surfaces}

In order to consider grid embeddings of graphs in non-planar surfaces, we need to give an appropiate representation of a surface of genus $n$. Of course, the first attemp to achieve that goal must be to use the well-known Classification of Surfaces (see, for instance [2]).

Theorem 1. [2] Every closed, connected, orientable surface is homeomorphic to one of the standard polygonal surfaces.

The standard polygonal surfaces are obtained by identifying the sides of a $4 n$-gone following the scheme described in Figure 1.

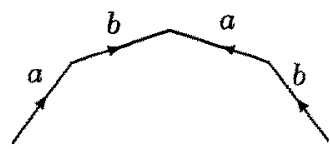

Fig. 1. One of the handles in a surfaces of genus $n$.

On the other hand, we can observe that in our problem the existence of an orthogonal system is fundamental. Nevertheless, the standard polygonal representation of surfaces lacks that system. However, it is possible to give another representation of surfaces. As a consequence of Theorem 1 we get the following classification theorem.

Theorem 2. Every closed, connected, orientable surface is homeomorphic to one of the standard orthogonal surfaces.

Where the standard orthogonal surface of genus $n$ is obtained by identifying the sides of a rectangle with $n-1$ small rectangles deleted in its interior following the scheme described in Figure 2.

Observe that in the standard orthogonal surface of genus $n$ there exist two distinguish directions (horizontal and vertical, those directions parallel to the 


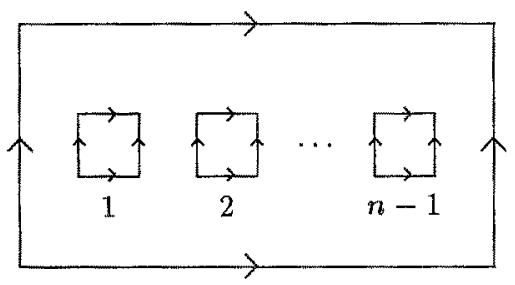

Fig. 2. The standard orthogonal surface of genus $n$.

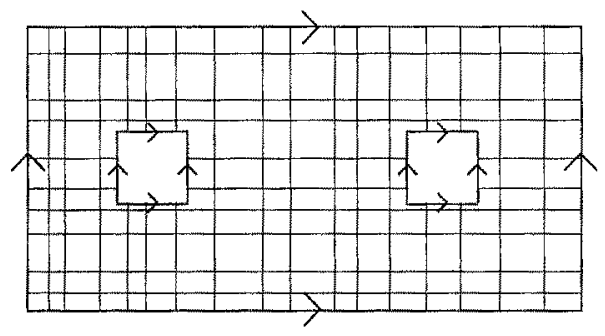

Fig. 3. A grid of the surface of genus 3 .

sides of the external rectangle). The horizontal direction will be called the direction of the parallels and the vertical direction will be called the direction of the meridians. Thus, any set of parallels and meridians will be called a grid of the standard orthogonal surface. Figure 3 shows an example.

Now, it is possible to say that a grid drawing (or embedding) of a graph in a surface of genus $n$ maps each edge to a chain of segments parallel to the sides of the external rectangle in the standard orthogonal surface and no two edges intersect except at a common vertex. A change of direction (from a parallel to a meridian) in an edge is called a bend. A rectilinear drawing is a grid drawing where each edge is either a horizontal or a vertical segment (i. e. a grid embedding without bends).

Figure 4 shows a grid drawing of a graph on the standard orthogonal surface of genus 3 .

As usual (see [5]), it is possible to identify an embedding with a rotation system (a cyclic permutation of the edges incident to each vertex) and two embeddings are said to be equivalent if their rotations systems are the same up to cyclic permutation. Two embeddings are said to be essentially equivalent if the two subgraphs constituted, one with the blocks with essential cycles (those "wrapping around" the surface or, more precisely, those homotopically non-trivial) and the other with the blocks without essential cycles are pairwise equivalent. Figures 5 and 6 show some examples. 


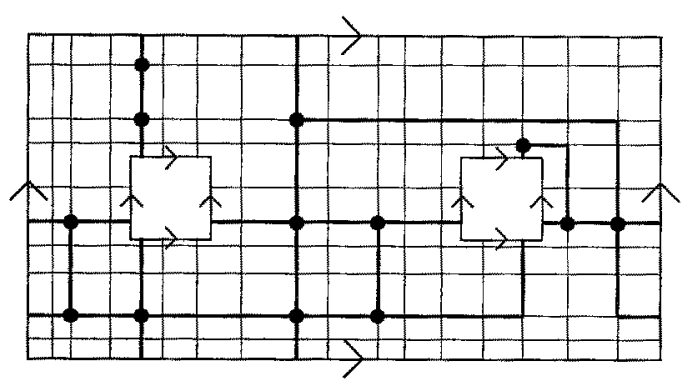

Fig. 4. A grid drawing of a graph on the standard orthogonal surface of genus 3 .

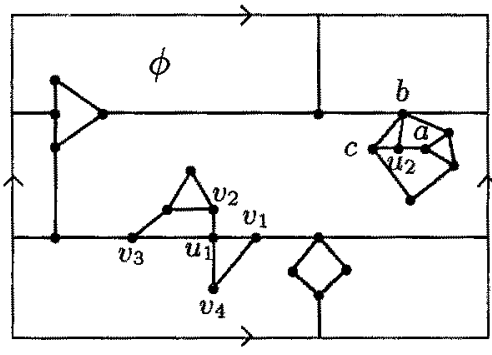

(a)

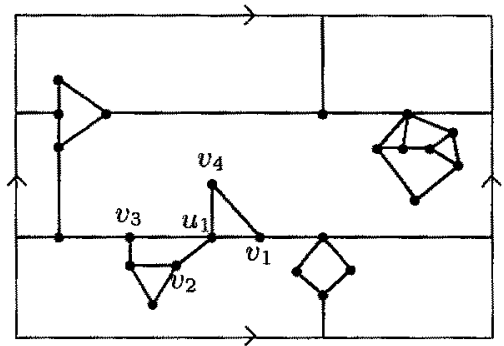

(b)

Fig. 5. (a) $\phi$ is an embedding on the torus of a graph with two blocks, one with essential cycles and the other without it. (b) This embedding is not essentially equivalent to $\phi$ because the embedding of the block with essential cycles is not equivalent.

\section{Essentially equivalent rectilinear drawing}

In this section, we prove that the following problem is NP-complete:

ESSENTIALLY EQUIVALENT RECTILINEAR DRAWING (EERD):

INSTANCE: Surface $S$, graph $G$, embedding $\phi: G \rightarrow S$.

QUESTION: Does there exist a rectilinear drawing of $G$ in $S$ essentially equivalent to $\phi$ ?

Theorem 3. EERD is NP-complete.

Proof. (SKETCH) EERD is easily seen to be in NP. For the second part of the proof, we transform 3-SATISFIABILITY (3SAT) to EERD. Let $U=\left\{u_{1}, u_{2}, \ldots, u_{n}\right\}$ be a set of variables and $C=\left\{c_{1}, c_{2}, \ldots, c_{m}\right\}$ be a set of clauses making up an arbitrary instance $\mathcal{S}$ of $3 \mathrm{sAT}$. Starting from $\mathcal{S}$, we construct an embedding $\phi_{\mathcal{S}}$ of a graph $G_{\mathcal{S}}$ in a suitable surface, as follows. We associate to each clause the 


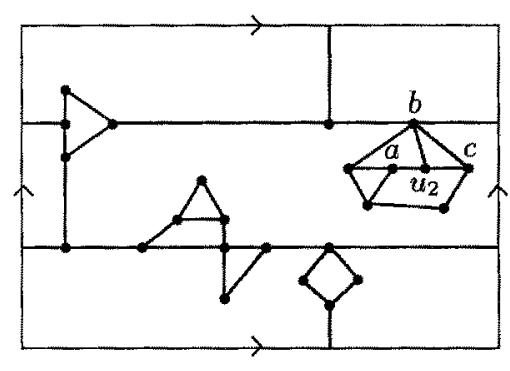

(a)

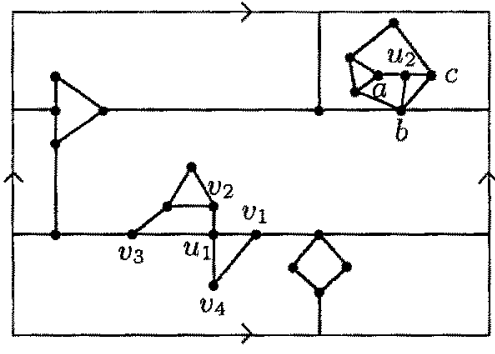

(b)

Fig. 6. (a) This embedding is not essentially equivalent to $\phi$ of Figure 5.(a) because the embedding of the block without essential cycles is not equivalent. (b) This embedding is essentially equivalent to $\phi$ of Figure 5 ,(a).

embedding of the graph shown in Figure 7, where each subgraph despicted in Figure 8 represents one of the three literals of the clause.

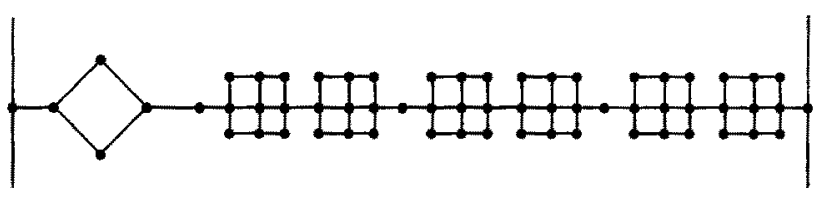

Fig. 7. Embedding associated to each clause.

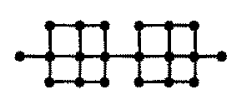

Fig. 8. Embedding associated to each literal.

Finally, we join the literals of the different clauses as it is shown in Figure 9, and we add enough handles to avoid crossings. (Figure 10 shows a complete example for the set of variables $U=\left\{u_{1}, u_{2}, u_{3}, u_{4}, u_{5}\right\}$ and the clauses $C=$ $\left\{c_{1}, c_{2}, c_{3}\right\}$ with $c_{1}=\left\{u_{2}, u_{3}, u_{5}\right\}, c_{2}=\left\{u_{1}, \overline{u_{4}}, u_{5}\right\}$ and $\left.c_{3}=\left\{u_{2}, u_{3}, \overline{u_{5}}\right\}\right)$. 


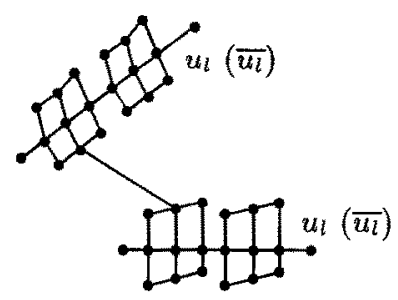

(a)

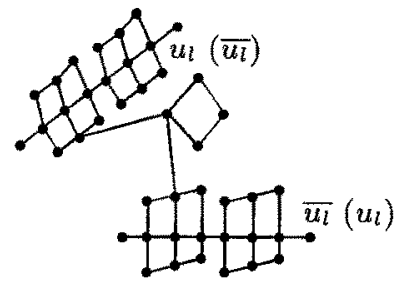

(b)

Fig. 9. (a) Union between embeddings associated to equal literals. (b) Union between embeddings associated to opposite literals.

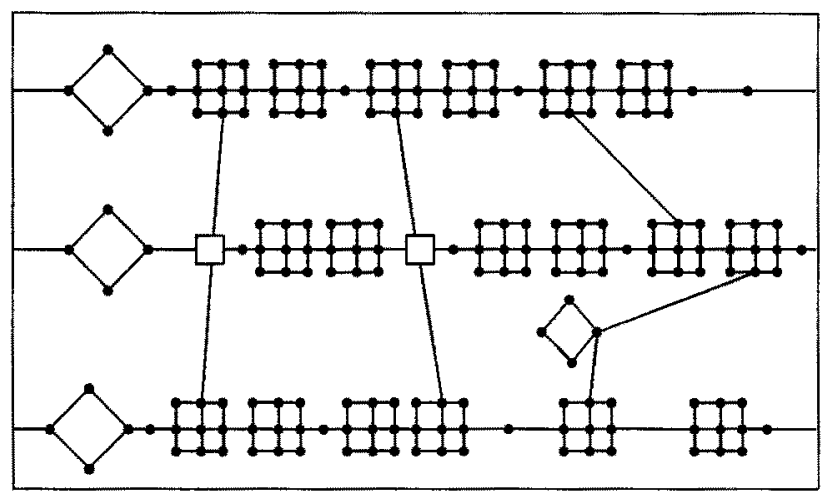

Fig. 10. An example for three clauses.

We claim that $\mathcal{S}$ is satisfiable if and only if there exists a rectilinear drawing of $G_{\mathcal{S}}$ essentially equivalent to $\phi_{\mathcal{S}}$.

In a rectilinear drawing of $G_{\mathcal{S}}$ essentially equivalent to $\phi_{\mathcal{S}}$, we say that one of the subgraph associated to a literal of a clause is vertical if the two vertices that have degree one in the subgraph are in the same meridian. It is horizontal if they are in the same parallel (those are the only possible options). Then, it is easy to see the following two facts:

1. Two literals corresponding to the same variable in different clauses are one of them vertical and the other horizontal if and only if one is the negation of the other.

2. In all graphs corresponding to clauses of $\mathcal{S}$ there exists at least one vertical subgraph associated to one of its literals (this happens because in all rectilinear embeddings of the graph associated to a clause, the subgraph that 
is not part of a literal has its vertices of degree one at different heights, see Figure 11).

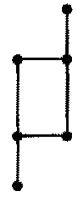

(a)

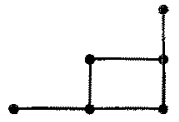

(b)

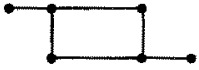

(c)

Fig. 11. Rectilinear embeddings of the graph associated to a clause.

Therefore, if we assign the value True to vertical literals and False to horizontal ones we get a satisfying truth assignment for $\mathcal{S}$.

Reciprocally, the assignation given above leads to a rectilinear drawing of $G_{\mathcal{S}}$ from a satisfying truth assignment for $\mathcal{S}$. In such a way that true literals correspond to vertical subgraphs, those vertical subgraphs are always drawn upward if we follow the graph representing the clause from left to right as it is shown in Figure 12.

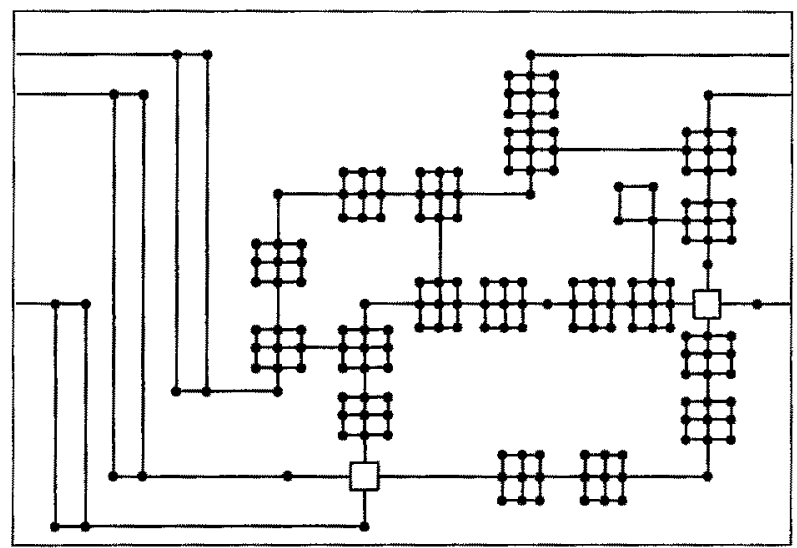

Fig. 12. Rectilinear embedding of the graph in the Figure 6.

As a consequence of Theorem 3 , the corresponding optimization problem related with EERD is NP-hard, then 
Corollary 4. Given an embedding $\phi: G \longrightarrow S$ of a graph $G$ in a surface $S$, computing a grid drawing of $G$ in $S$ essentially equivalent to $\phi$ with the minimum number of bends is NP-hard.

\section{Rectilinear drawing of graphs}

As it has been said in the introduction, Garg and Tamassia [4] proved that testing whether a graph is rectilinear planar is an NP-complete problem. In this section we study a problem related in some way with that, which we can establish in the following terms.

RECTILINEAR DRAWING IN SURFACE (RDS):

INSTANCE: Graph $G$.

QUESTION: Does there exist a surface $S$ and a rectilinear drawing of $G$ in $S$ ?

Theorem 5. RDS is NP-complete.

Proof. (SKeTCh) Again, RDS is easily seen to be in NP. For the second part of the proof, we will transform the following well-known NP-complete problem: NOT-ALL-EQUAL-3SAT (NE3SAT):

INSTANCE: Set $U$ of variables, collection $C$ of clauses over $U$ such that each clause $c \in C$ has $|c|=3$.

QUESTION: Is there a truth assignment for $U$ such that each clause in $C$ has at least one true literal and at least one false literal?

In the proof of Theorem 3, we used the fact that in any rectilinear embedding of a given graph, there are two points that we know that are not over the same parallel, here we will exploit that in any vertex of degree 3 in a rectilinear embedding at least one of its incident edges must be vertical and at least one of its incident edges must be horizontal.

Let $U=\left\{u_{1}, u_{2}, \ldots, u_{n}\right\}$ be a set of variables and $C=\left\{c_{1}, c_{2}, \ldots, c_{m}\right\}$ be a set of clauses making up an arbitrary instance $\mathcal{S}$ of NE3SAT. Starting from $\mathcal{S}$, we construct a graph $G_{\mathcal{S}}$ in the following way:

Each variable $u_{i}, i=1, \ldots, n$ is represented by a sequence of cycles $C_{3}$ of length 3 adjacent by different vertices to the its anterior and to its posterior. The cycles in an odd position in the sequence will represent the variable in its affirmative form and those in even position will represent the variable in its negative form. The length of this sequence is determined by the number of literals where the variable appears in such a way that we will have enough vertices of degree 2 to join with new vertices that will represent the clauses (increasing, in this way the degree of some of those vertices of degree 2 up to degree 3 ). Then vertices representing clauses will have degree 3 (Figure 13 shows the graph corresponding to the set of variables $U=\left\{u_{1}, u_{2}, u_{3}, u_{4}\right\}$ and the clauses $C=\left\{c_{1}, c_{2}, c_{3}\right\}$ with $c_{1}=\left\{u_{1}, \overline{u_{2}}, u_{4}\right\}, c_{2}=\left\{\overline{u_{1}}, u_{3}, u_{4}\right\}$ and $\left.c_{3}=\left\{u_{2}, u_{3}, u_{4}\right\}\right)$.

Observe that in a rectilinear drawing of $G_{\mathcal{S}}$ it is easy to check the following three facts:

1. A subgraph associated to a literal is always either vertical or horizontal. 


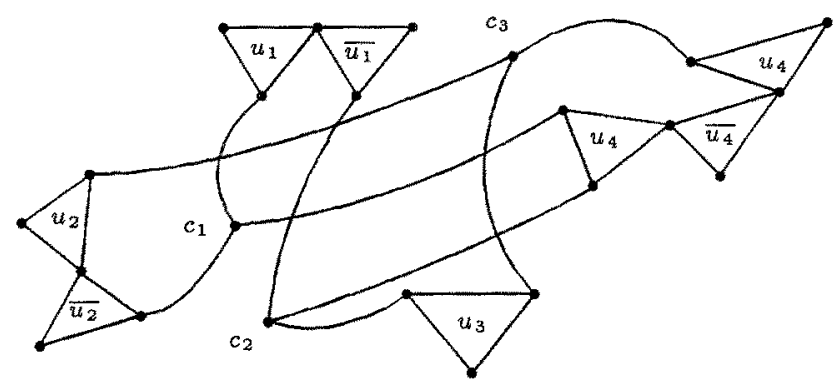

Fig. 13. An example for three clauses.

2. In the case that two literals correspond to the same variable, one of them is vertical and the other is horizontal, if and only if one is the negation of the other.

3. Each vertex corresponding to a clause is adjacent to at least one vertical literal and at least to one horizontal literal.

Therefore, if we assign the value True to vertical literals and False to horizontal ones we get a satisfying truth assignment for $\mathcal{S}$.

Reciprocally, the assignation given above leads to a rectilinear drawing of $G_{\mathcal{S}}$ from a satisfying truth assignment for $\mathcal{S}$. In such a way that true literals correspond to vertical subgraphs (see Figure 14).

As above, the corresponding optimization problem is NP-hard

Corollary 6. Given a graph $G$, finding a surface $S$ and an embedding $\phi$ of $G$ in $S$ such that $\phi$ minimizes the number of bends among all embeddings in any surface of $G$ is NP-hard.

\section{Conclusions and Open Problems}

In this paper we give an adequate model for studying embeddings of graphs in the grid of non-planar surfaces, proving, moreover, that two natural problems arising in such embeddings are NP-complete problems, namely, testing whether a graph admits a rectilinear (without bends) embedding essentially equivalent to a given embedding; and given a graph, testing if there exist a surface such that the graph admits a rectilinear embedding in that surface. Hence, the corresponding optimization problems are NP-hard.

Of course, the main open problem related to our work is deciding if testing whether a graph admits a rectilinear embedding equivalent (without the restriction of being essentially equivalent) to a given embedding is an NP-complete 


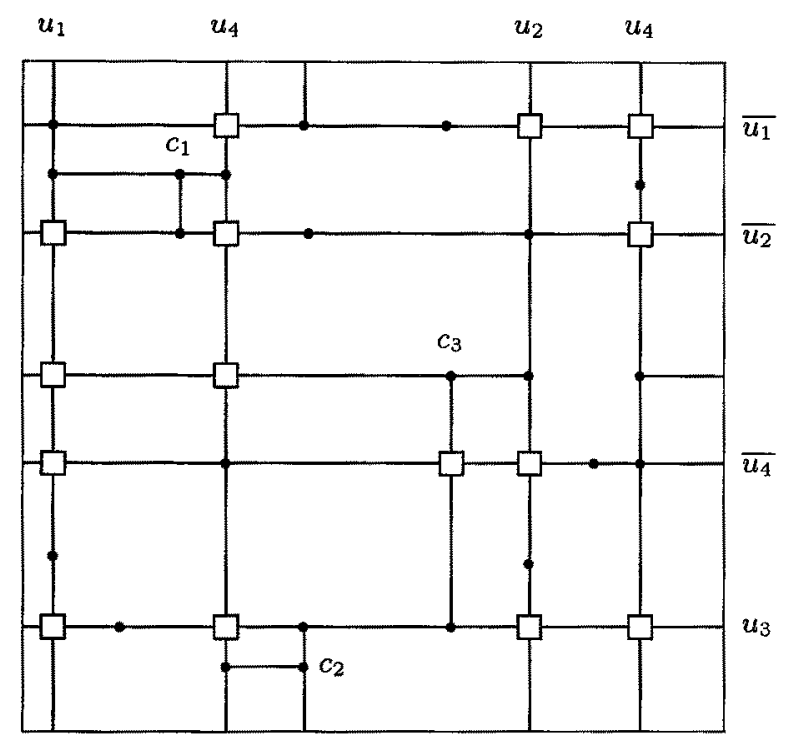

Fig. 14. Rectilinear embedding of the graph in the Figure 9.

problem. Moreover, it can be interesting to study the existence of approximations to the optimal solutions of the corresponding optimization problems.

\section{References}

1. D. Dolev and R. Trickey. On linear area embedding of planar graphs. Technical Report STAN-CS-81-876, 1981.

2. M. Fréchet and Ky Fan. Initiation to Combinatorial Topology. Weber and Schmidt, 1967.

3. M. R. Garey and D. S. Johnson. Computers and Intractability: a guide to the theory of NP-completeness. Freeman, 1979.

4. A. Garg and R. Tamassia. On the computational complexity of upward and rectilinear planarity testing. In R. Tamassia and I. G. Tollis, editors, Graph Drawing 94, Lectures Notes in Computer Science, pages 286-297. Springer-Verlag, 1994.

5. J. L. Gross and T. W. Tucker. Topological graph theory. John Wiley \& Sons, 1987.

6. T. A. J. Nicholson. Permutation procedure for minimizing the number of crossings in a network. Proc. Inst. Elec. Eng., 115:21-26, 1968.

7. R. Tamassia. On embedding a graph in the grid with the minimum number of bends. Siam J. Comput., 16(3):421-444, 1987.

8. C. Thomassen. The graph genus problem is NP-complete. Journal of Algorithms, 10:568-576, 1989. 